\title{
El médico compasivo es el mejor "medicamento" para el enfermo
}

\author{
The compassionate doctor is the best "medicine" \\ for the patient
}

Emiliano Paico-Vílchez '

Paico-Vílchez E. El médico compasivo es el mejor “medicamento" para el enfermo. Rev Soc Peru Med Interna. 2020;33(4): I87-I9I. https://doi.org/10.36393/spmi.v33i4.570

\section{RESUMEN}

Las reflexiones que hace el autor son lo suficientemente paradigmáticas para señalar lo siguiente:

El médico compasivo consigue un efecto de sanación significativamente mayor que cuando no practica el sentimiento de la compasión.

El médico compasivo es el mejor "medicamento" del enfermo, ya que éste se beneficia con los óptimos resultados en su salud; asimismo, con la experiencia de satisfacción de haber sido tratado con cariño, solidaridad, respeto, humildad, sinceridad, honradez, comprensión y compasión; es decir, ser tratado con espíritu humanitario.

Actuando el médico con compasión también sale beneficiado, pues brinda atención médica efectiva y más diligente, tiene más credibilidad, mejora la relación médico paciente, tiene menos agotamiento y evita incurrir en mala praxis.

Palabras clave: Médico compasivo - Mejor medicamento - Enfermo

\section{ABSTRACT}

The reflections made by the author are paradigmatically sufficient to point out the following:

The compassionate medic achieves a significantly greater healing effect than when he does not practice the feeling of compassion.

The compassionate doctor is the best "medicine" for the patient, because he benefits from the optimal results in his health; also, with the experience of satisfaction of having been treated with affection, solidarity, respect, humility, sincerity, honesty, understanding and compassion; that is, to be treated in a humane spirit.

Acting the doctor with compassion also benefits himself, as he provides effective and more diligent medical care, has more credibility, improves the doctor-patient relationship, has less exhaustion and avoids incurring malpractice.

Key words: Compassionate doctor - Best medicine - Sick

Cirujano pediátrico, profesor de la Universidad Privada Antenor Orrego y ex jefe del Servicio de Cirugía Pediátrica y del Servicio de Especialidades Quirúrgicas del Hospital Belén de Trujillo, Perú.
"Si compadeces en algo, padeces, y médico que compadece mejor medicina ofrece”. Aforismo popular

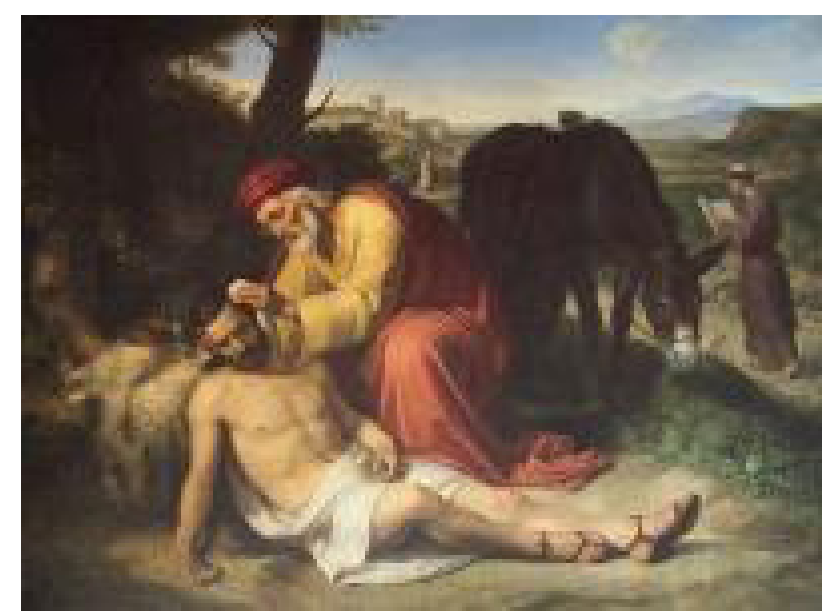

El buen samaritano. Óleo sobre tela. Pelegrí Clavé i Roquer (I8I I-I880). 


\section{INTRODUCCIÓN}

Quiero iniciar este artículo manifestando que no todo lo que brilla es oro, ni todo lo que tiene etiqueta de buen médico es buena persona; pues, para ser buen médico, en el sentido más estricto de la palabra, tiene que necesariamente ser una buena persona. No debemos confundir a un médico competente con un médico que es buena persona, es decir un buen médico.

El medico competente es aquel que posee la habilidad para diagnosticar, tratar, curar o mejorar una enfermedad. En cambio, el buen médico no solo es competente en tratar la enfermedad sino que, esencialmente, también es competente en tratar a la persona enferma.

Ser buena persona incluye un conjunto de conocimientos, conductas, actitudes y motivaciones tan extenso que es difícil de delimitarlo. Pero, no sólo es difícil la delimitación sino que, además, es complicado formular una definición que trascienda todas las culturas ya que, es probable, lo que llamamos ser una buena persona en una cultura determinada no lo sea en otra cultura diferente, incluso en una misma cultura.

Sin embargo, existen rasgos o virtudes que identifican a una buena persona; por ejemplo: la bondad, solidaridad, respeto, humildad, sinceridad, confianza, honradez, empatía, compasión, etc.

Entre las virtudes citadas, la que más caracteriza a un buen médico es la compasión. La compasión va más allá que la empatía, es más intensa. Mientras que la empatía del médico es la capacidad de compartir o sentir la enfermedad del paciente, la compasión del médico es la capacidad de no sólo compartir o sentir la enfermedad sino que siente obligación de aliviar el sufrimiento que causa la enfermedad, desea contribuir en el bienestar del paciente. Por esto se dice que la compasión es la sublimación de la empatía.

Por otra parte, si bien es cierto que el extraordinario progreso logrado por la ciencia y la tecnología médica permite abolir o aliviar el sufrimiento de la enfermedad mejorando e incrementando la calidad de vida, también es cierto que este progreso ha devenido como un búmeran al provocar cambios en la atención del paciente, pues su carácter personal e íntimo que ha tenido en el trascurso de la historia de la medicina resulta ahora, por el contrario, impersonal y fragmentado. Estos cambios, igualmente, han traído como consecuencia la pérdida del liderazgo del médico y el deterioro significativo de la relación médicopaciente que ha sido siempre piedra angular del acto médico.

Karl Jaspers, en su obra La práctica Médica en la Era Tecnológica, señala lo siguiente: "En la medicina moderna, todo parecería estar en el mejor de los órdenes. Día a día se logran grandes resultados. Pero, lo asombroso es que en los enfermos y en los médicos aumenta la insatisfacción". ${ }^{1}$ Esto que escribió el doctor Jaspers hace más de 70 años, actualmente está vigente, probablemente debido a nuestra apatía o tal vez por encontrarnos desarmados para bregar el cambio que lleve a evitar los perjuicios del mal uso de la tecnología. Si persiste la indiferencia, los problemas actuales continuarán y los pacientes seguirán siendo los perjudicados.

Teniendo en consideración las reflexiones sugeridas de los párrafos precedentes, es imperativo abordar un tema por demás relevante como es la compasión que debe tener todo médico, sentimiento que se convierte en una herramienta que adquiere un valor esencial, por no decir imprescindible, en la relación médico paciente.

\section{RELACIÓN MÉDICO - PACIENTE}

La relación médico paciente es aquella que se establece entre dos personas, el enfermo que busca ayuda y el médico que puede ofrecérsela. Es un encuentro entre dos personas con necesidades complementarias: la persona enferma que sufre y el médico que dispone de herramientas para tratar la enfermedad.

En esta relación, el paciente no es un mero objeto de la atención clínica sino el sujeto que la justifica y da sentido. Se trata de una relación "casi diádica y asimétrica" en la que sus integrantes tienen derechos y obligaciones basados en el respeto que implica autonomía, justicia, confidencialidad y veracidad. ${ }^{2}$

Juan Gérvas y col refieren que "en realidad todo encuentro es sagrado, en el que el paciente confía en su médico, y expone su piel y su alma en unos minutos de entrevista. El médico tiene el consentimiento tácito para explorar el campo abierto e ir más allá de la superficie corporal y del brillo espiritual." 3

Sin embargo, como mencionan Miles y Loughlin y Miles y Mezzich, referidos por Stephen Post, muchos pacientes se quejan del tratamiento impersonal y deshumanizante, tanto así que a menudo se hace sentir a los pacientes como "la hernia de la cama 2" o "la vesícula de la cama 14", en lugar de personas con un nombre, con una historia única de enfermedad, $s$ in respetar su dignidad humana. ${ }^{4}$

Los pacientes, además de pedir un tratamiento efectivo para su enfermedad, buscan en los médicos cariño y compasión, en ocasiones ignorando ellos mismos que es una de las principales cosas que están buscando.

En 1996, un equipo de expertos en bioética del Hastings Center de Nueva York, refiriéndose a los fines de la medicina manifiesta que "las personas suelen recurrir a la medicina porque se encuentran mal, han sufrido alguna lesión o padecen una discapacidad mental o física. El médico, por su parte, responde buscando la causa del mal, suponiendo que podrá encontrarla en un órgano o miembro lesionado o enfermo. Cuando se da ese caso, el médico intenta curar el mal y hacer que el paciente recupere su antiguo estado de bienestar y el normal funcionamiento. Sin embargo, el paciente no suele presentar al médico su órgano enfermo, incluso si sabe o sospecha que ése es el origen de su malestar; el paciente busca más que la simple cura, busca comprensión y compasión." 5

Por eso, es importante que los médicos no debemos olvidar que el paciente es una persona como nosotros; entonces, debemos tratarlos como nos gustaría que nos 
tratasen a nosotros, a nuestros hijos, padres o hermanos si estuviésemos enfermos. Los pacientes tienen nombres, sentimientos, intimidades, proyectos, etc., y cada paciente vive de modo diferente su enfermedad. Si no entendemos cómo reaccionan los pacientes es que el conocimiento de ellos y de su contexto es insuficiente todavía; y, esto, no es ser un buen médico.

\section{MÉDICO COMPASIVO}

La compasión es un sentimiento humano que se manifiesta desde el contacto y la comprensión del sufrimiento de otro ser. Más intensa que la empatía, la compasión es la percepción y la compenetración en el sufrimiento del otro, y el deseo y la acción de aliviar, reducir o eliminar por completo tal situación dolorosa.

Ser compasivo es un sentimiento inherente al ser humano. Los seres humanos nacen con la capacidad de sentir compasión, y los involucrados en el cuidado de los pacientes y sus familias pueden aprender a profundizar su capacidad para brindar atención compasiva en cada interacción en la que se les solicita. ${ }^{6}$

La compasión va más allá de la empatía, pues ésta es una forma de inteligencia, de capacidad cognitiva, de "entender inteligentemente" mientras la compasión se refiere a un nivel más básico, de solidaridad ante el sufrimiento. La empatía es más de la corteza cerebral, en cambio la compasión es más del sistema límbico. La compasión es un sentimiento, no un conocimiento. ${ }^{7}$

La compasión es un sentimiento indispensable en el ejercicio de la medicina. Los médicos que no son compasivos no satisfacen las necesidades de los pacientes. Numerosos estudios revelan que cuando los médicos tratan o asisten a los pacientes con compasión, estos con frecuencia se sanan antes, sienten menos dolor e intensidad, e incluso se recuperan con más rapidez.

Los doctores Stefano Alice y Mara Fiorese refieren que, "Si se revisara exhaustivamente la literatura cientifica, podría advertirse que hay algo capaz de disminuir el dolor posquirúrgico, aumentar las tasas de supervivencia de los pacientes con cáncer, reducir el índice de mortalidad de los pacientes cardiacos de alto riesgo y recortar el índice de hospitalización de los pacientes diabéticos. Como si esto no fuera suficiente, también puede mejorar el sistema inmunitario." ${ }^{8}$ Ellos continúan diciendo: "Esa misteriosa sustancia es la compasión, o mejor aún los médicos compasivos."

El doctor James Doty, fundador y director del Centro de Investigación y Educación sobre la Compasión y el Altruismo de la Facultad de Medicina de la Universidad de Stanford, afirma que "Cuando la atención de la salud se ofrece con amabilidad y compasión, tiene un efecto significativamente mayor que cuando se ofrece de una forma desapasionada que da por sentado que la conexión humana no tiene un beneficio". 9 Aunque la medicina tiene el poder de curar, dijo, la forma en que se administra puede hacer una inmensa diferencia, no solo para los pacientes sino también para los médicos.
El mismo doctor Doty, revisando la literatura encontró investigaciones que revelaban que un tratamiento compasivo ayudaba no solo a reducir el dolor y la ansiedad sino que, también, se vinculaba con una presión arterial más baja, unas estadías más cortas en el hospital, e incluso reducía la gravedad y la duración del resfriado común.

Por su parte el doctor Beth Lown y col, en los meses de setiembre y octubre del 2010, hicieron una encuesta a 800 pacientes hospitalizados y a 510 médicos, a quienes le les leyeron una descripción de la atención médica compasiva y sus implicancias. Los resultados fueron los siguientes: Tanto los pacientes como los médicos estuvieron de acuerdo en que los resultados de salud dependen no solo de las habilidades médicas y el conocimiento del médico sino también de su comunicación eficaz y apoyo emocional.

De los encuestados que dijeron que entendían la descripción de la atención compasiva, el 85 por ciento de los pacientes y el 76 por ciento de los médicos dijeron que es "muy importante" para el éxito del tratamiento médico. Además, el 81 por ciento de los pacientes y el 71 por ciento de los médicos estuvieron de acuerdo en que la buena comunicación y el apoyo emocional pueden marcar la diferencia en si un paciente vive o muere. Solo el 19 por ciento de los pacientes y el 23 por ciento de los médicos dijeron que las habilidades médicas y el conocimiento científico del médico son los únicos factores que determinan si un paciente vive o muere. ${ }^{10}$ Los pacientes y los médicos en general estuvieron de acuerdo en la importancia de muchos elementos del cuidado compasivo. Ambos grupos dijeron que los elementos más importantes eran ser tratados con respeto, comunicación clara de información, comunicar los resultados de las pruebas de manera oportuna y sensible, ser tratado como una persona y no solo una enfermedad, ser escuchado, participar en las decisiones de tratamiento y confiar sus médicos.

Por lo mencionado, se puede decir que existe evidencia sólida de que los médicos compasivos mejoran los resultados de salud y las experiencias de atención de los pacientes; por lo tanto, es imperativo que todos los que nos dedicamos a tratar enfermos debemos hacerlo practicando ese noble sentimiento llamado compasión.

Si el médico desea tratar o asistir con compasión a un paciente es necesario que integre cualidades intrínsecas de su profesión, que incluyen sus propios valores y creencias, con lo que podríamos llamar habilidades esenciales para el cuidado compasivo. Cuando hablamos de integrar las cualidades intrínsecas del profesional nos estamos refiriendo a aquellas que son propias de él mismo, a aquello que hace que cada uno sea de una manera distinta a los demás. El paciente detecta inmediatamente esa propiedad fundamental de tener delante a alguien auténtico, a alguien que quiere ayudar, a alguien que no finge, a alguien que no está impostado o que no está intentando demostrar nada. Allí hay simplemente una persona, en este caso un profesional, que lo que quiere es ayudar. ${ }^{10}$

El médico compasivo que aspira colaborar en la sanación de su paciente se acerca con su propio y auténtico modo 
de ser, y fomenta habilidades sentimentales para una mejor relación médico-paciente, haciéndole notar que está interesado en él como ser humano (cuerpo y alma) y que quiere ayudarlo; para ello es necesario tener en cuenta las recomendaciones que hace el doctor Carlos Centeno de la Facultad de Medicina de la Universidad de Navarra de España, a quien seguimos de cerca en este tema. ${ }^{11}$

El médico debe, en primer lugar, escuchar atentamente los detalles que le manifiesta el paciente; debe interpretar el tono de sus palabras, el silencio, los mensajes implícitos. Debe intuitivamente comprender profundamente las necesidades del paciente. Se dice que esta escucha intuitiva exige un sentimiento de instrumento en el médico, sentirse catalizador de un proceso y la ausencia de un afán de protagonismo en todo el cuidado. Cuando escuchamos así, delicadamente, llegarán a nosotros frases que expresan sufrimiento, frases del estilo de porqué yo, porqué a mí, no lo entiendo, no quiero morir. ${ }^{11}$

En segundo lugar, tiene que mirar con ternura. La mirada del médico que quiere sanar debe ser una mirada pletórica de amor. Es una mirada que busca más allá del paciente. Que busca la esencia de su persona y que supone su aceptación incondicional y de sus problemas. Tenemos que mirar lo que no se ve. A observar las características inseparables de la espiritualidad. A captar la chispa divina que hay en todo enfermo. La mirada compasiva es una mirada protectora, que extiende un manto compartido y hace que el paciente no se sienta un extraño frente al médico sino parte de la familia compasiva. ${ }^{11}$ Es una mirada que dice: entiendo lo que siente y padece, no me cansa oírle, soy solidario con su vulnerabilidad, además de médico soy persona, cuente conmigo. $^{7}$

En tercer lugar, junto con escuchar y mirar, está la capacidad de intervenir con la conversación. Es decir, el médico debe tener la capacidad de hablar con el paciente en el momento oportuno, teniendo en cuenta de no herir susceptibilidades. Los que confían mucho en su capacidad de comunicación tienden a bloquear el proceso personal del paciente: sofocan el flujo de conversaciones terapéuticas que los propios pacientes podrían haber iniciado. Cuando las palabras son necesarias para el cuidado existencial del enfermo, para su sanación, lo fundamental es decir poco y con sensatez. No decir palabras inapropiadas para no "meter la pata". ${ }^{11}$

En cuarto lugar, el médico debe cultivar la capacidad de acercarse físicamente al paciente. Nos referimos al contacto empático, por ejemplo: saludar con afecto, sostener la mano de un niño asustado mientras están curándole una herida, coger la mano delicadamente mientras se mantiene una conversación terapéutica o en momentos de emoción, un toque en el hombro o una presión suave en el brazo para acompañar una mala noticia. Este contacto con el paciente señala que hay intimidad suficiente para abordar los temas más serios. El cuidado físico del paciente, el cuidado de su cuerpo, tiene efecto más allá de lo físico y afecta a su bienestar. Por eso, debemos tener conciencia de la capacidad terapéutica de acciones físicas capaces de materializar el cuidado espiritual del enfermo. El modo de presentarnos, el arreglo personal, la actitud del médico que entra a la habitación del paciente para examinarlo, o la palpación suave en la zona dolorosa durante el examen físico, etc. puede ser una extraordinaria colaboración en el proceso interior del enfermo. ${ }^{11}$

La capacidad de ayudar a sanar en aspectos profundos se resume en la presencia terapéutica del médico. El médico se acerca con francos deseos de ayudar al paciente. El paciente percibe esa presencia autentica, nota enseguida que allí está no solo su médico sino alguien que viene a ayudar, que quiere ayudarlo personalmente poniendo en juego su propia persona. Además, es presencia como sujeto vulnerable, no solo como médico sino como alguien al que le afecta lo que le ocurre al paciente y que muchas veces también se conmueve. El sentirse vulnerable, afectado por el sufrimiento del paciente, algo hay de humanidad compartida. Pero al médico no le importa que le afecte, porque en la medida que esto ocurre está ayudando más al paciente. ${ }^{11}$

Como se puede notar, para ser un médico compasivo se requiere tener una personalidad equilibrada y sensata, ser afectuoso y empático, ser humilde y solidario, ser voluntarioso y perseverante, y tener disposición al sacrificio. Con todas estas virtudes se logra ser un genuino médico compasivo.

Finalmente, se debe subrayar que, siendo la compasión parte esencial en la sanación de los pacientes, es ineludible que los médicos cultiven este noble sentimiento, tanto en su formación médica inicial como en su formación permanente; $\mathrm{y}$, que la compasión sea considerada y evaluada como una competencia central en proveedores de salud.

Asimismo, siendo la compasión parte de la respuesta clínica de calidad, como tal debería incluirse, de manera reglada, en el currículo formativo de las facultades de medicina, con la finalidad que los estudiantes desde sus prácticas preprofesionales sean empáticos y compasivos con los pacientes.

\section{CONCLUSIONES}

La compasión es un sentimiento mediante el cual una persona siente el mismo sufrimiento al ver a otra persona que está sufriendo, y que impulsa a aliviar el dolor o sufrimiento, a remediarlo o a evitarlo en la medida de lo posible.

El médico compasivo consigue un efecto de sanación significativamente mayor que cuando no actúa con compasión.

La atención médica compasiva constituye el mejor "medicamento" del enfermo, pues éste se beneficia con óptimos resultados en su salud, con la experiencia de satisfacción de haber sido tratado con cariño, solidaridad, respeto, humildad, sinceridad, honradez, comprensión y compasión; es decir, ser tratado con espíritu humanitario.

Existen tips o pautas que permiten comprender mejor las necesidades del paciente y la situación de vulnerabilidad que tiene, lo que permite convertirse en un médico compasivo; y, con este sentimiento de compasión, puede 
ayudar eficientemente al paciente y a sus familiares, en todo lo que sea posible, para aliviar el sufrimiento.

El médico debe cultivar la actitud de compasión, tanto en su formación médica inicial como en su formación permanente. Actuando con compasión también sale beneficiado, pues brinda atención médica efectiva y más diligente, tiene más credibilidad, mejora la relación médico paciente, tiene menos agotamiento y evita incurrir en mala praxis.

\section{REFERENCIAS BIBLIOGRÁFICAS}

I. Jaspers K. La práctica médica en la era tecnológica. Barcelona: Gedisa; 1988.

2. Vidal y Benito María del Carmen. La relación médico paciente: Bases para una comunicación a medida. Buenos Aires: Lugar Editorial; 2010.

3. Gérvas Juan, Pérez Fernández Mercedes, Gutiérrez Parres Blanca. Consultas sagradas: serenidad en el apresuramiento. Aten Primaria. 2009; 4 I (I):4I-44

4. Post S. Compassionate care enhancement: benefits and outcomes. The International Journal of Person-Centered Medicine. 20 I I; I (4): 808813.
5. Gutiérrez Fernández, Rodrigo. La Humanización de (en) la Atención Primaria. Rev Clin Med Fam. 2 I 07; I ( I).URL disponible en: http://scielo. isciii.es/scielo.php?script=sci_arttext\&pid=SI699-695X2017000 100 005

6. Klimecki OM, Leiberg S, Lamm C, Singer T. Plasticidad neural funcional y cambios asociados en el afecto positivo después del entrenamiento de compasión. Cereb Cortex. 20 I 3; 23: I 552-I56I. doi: 10.1093 / cercor / bhs |42. [PubMed] [Cross Ref] [Google Scholar] Vidal y Benito, M.C. (2002) Acerca de la buena comunicación en medicina. Buenos Aires, CEMIC.

7. Gérvas Juan. Mostrar compasión con los pacientes no es una opción sino una exigencia ética. Acta Sanitaria, Madrid. 29 junio, 2019. URL disponible en: https:/www.actasanitaria.com/mostrar-compasioncon-los-pacientes-no-es-una-opcion-sino-una-exigencia-etica/

8. Alice Stefano, Fiorese Mara. Compasión: Qué es y por qué es importante en medicina. El Foro Económico Mundial, 26 abril 2017. URL disponible en: https://es.weforum.org/agenda/2017/04/ compasion-que-es-y-por-que-es-importante-en-medicina/

9. Doty Juan. La amabilidad y la compasión son la receta para una mejor atención de salud. Intra Med. 02 diciembre, 20I4. URL disponible en: https://www.intramed.net/contenidover.asp? contenidoid $=8582 \mathrm{l}$

10. Lown, BA, Rosen J and Marttila J. (20II). An agenda for improving compassionate care: a survey shows about half of patients say such care is missing. Health Affaire. 30(9): 1772-78.

II. Centeno Cortés, Carlos. Compasión: Corazón del ejercicio de la medicina. An Real Acad Med Cir Vall. 201 7; 54: 289-294.

Fecha de recepción: 27-I I-2020.

Fecha de aceptación: 04-12-2020. 